\title{
DESCRIÇÃO DOS FUNDAMENTOS TÉORICOS E PRÁTICOS DO BLOWDOWN APLICADOS AO ALTO FORNO 03 DA ARCELORMITTAL AÇOS PLANOS AMÉRICA DO SUL*
}

\author{
Filipe Sathler ${ }^{1}$ \\ Ramiro da Conceição do Nascimento Junior ${ }^{2}$ \\ Claudio César da Costa ${ }^{3}$ \\ Estefan Campos Ribeiro ${ }^{4}$
}

\begin{abstract}
Resumo
Mediante efeitos de sazonalidades do mercado Siderúrgico, períodos de crise, aliados às necessidades de reparos de equipamentos, os Altos Fornos são, eventualmente, submetidos ao desligamento. O procedimento específico mais utilizado é o Blowdown, com o abaixamento de carga até nível de ventaneiras, facilitando posterior limpeza e reparo. Para sucesso deste procedimento, é essencial um planejamento adequado das atividades. Informações técnicas e experiências operacionais permitiram o embasamento teórico e a execução do Blowdown do Alto Forno 03 da ArcelorMittal FCSA (Tubarão). Como preparação, foi efetuado o aumento do fluxo periférico (remoção de cascão) e o aumento da permeabilidade do Cadinho (maior circulação de líquidos, limpeza e drenagem). Realizou-se também a preparação de equipamentos específicos e controle dos parâmetros operacionais. $O$ abaixamento de carga foi devidamente realizado conforme planejado, até o nível de ventaneiras e permitindo paredes internas livres de cascões.
\end{abstract}

Palavras-chave: Siderurgia; Alto forno; Blowdown.

\section{THEORICAL AND PRACTICAL FUNDAMENTALS DESCRIPTION FOR BLOWDOWN ON BLAST FURNACE 03 OF ARCELORMITTAL FLAT CARBON SOUTH AMERICA}

\section{Abstract}

Through the effects of seasonality steelmaking market, periods of crisis, besides requirements of equipment repairs, Blast Furnaces are eventually submitted to prolonged stoppages. This activity can be accomplished by a specific procedure named as Blowdown, controlling burden descent until tuyeres for subsequent cleaning and repairs. Operational activities must be correctly planned and executed to guarantee successful Blowdown. Therefore, technical information and operational experiences established the theoretical concepts and plan for Blowdown execution at Blast Furnace 03 in ArcelorMittal FSCA (Tubarão). For preparation, it was improved peripheral flow (skull removal) and hearth permeability (liquids circulation and drainage). Also, it was made preparation of specific equipment and control of operating parameters. The burden lowering was appropriately performed as planned until burden reaches tuyeres elevation, and allowing efficient cleaning of inner walls.

Keywords: Steelmaking; Blast furnace; Blowdown.

1 Engenharia Metalúrgica, Especialista de Altos Fornos, Unidade Técnica da Área de Redução, ArcelorMittal Tubarão, Serra, Espírito Santo, Brasil.

2 Engenharia Metalúrgica e de Materiais, Pós-Doutorado, Professor, Departamento de Metalurgia e Materiais, Instituto Federal do Espírito Santo (IFES), Vitória, Espírito Santo, Brasil.

3 Engenharia Metalúrgica, Especialista de Altos Fornos, Unidade Técnica da Área de Redução, ArcelorMittal Tubarão, Serra, Espírito Santo, Brasil.

4 Engenharia Metalúrgica, Especialista de Altos Fornos, Unidade Técnica da Área de Redução, ArcelorMittal Tubarão, Serra, Espírito Santo, Brasil. 


\section{INTRODUÇÃO}

Ocasionalmente, faz-se necessário a realização de paradas de funcionamento do Alto Forno por vários dias, seja por condições de limitação de mercado ou para efetuar algumas atividades de Reforma Programada ou reparos diversos (Cadinho, Regeneradores, Staves, etc), segundo as condições de operação e vida útil. [1,4].

Nestas paradas de longa duração, tem-se procedimento específico de preparação de parada, com a execução de certas atividades para preservar a estrutura interna do forno. Dentre os diferentes métodos para realização do desligamento de um Alto Forno, o Blowdown tem sido o mais aplicado desde a década de 1970 [4,8,10,11].

O Blowdown consiste no abaixamento de carga e redução do volume de sopro até que o nível de carga alcance a altura das ventaneiras (Figura 1), propiciando um forno livre de cascão e o mais vazio possível (coque residual do Homem Morto e material líquido remanescente no cadinho, como mostrado na Figura 2), permitindo maior facilidade de inspeção das condições de equipamentos, partes internas do forno, vazamentos de água ou gás. Possibilita também maior rapidez de limpeza e reparos, sendo que o reinício de operação com o carregamento de material novo no topo acarreta em menos complicações de processo do que o retorno do forno após um "Abafamento" ou "Banking" (material fino/antigo/degradado no interior do forno podendo causar engaiolamento) $[1,4-6,10]$.

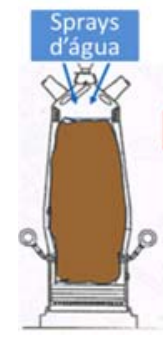

Figura 1. Evolução do procedimento de Blowdown. [10]

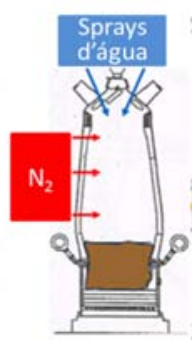

(1)

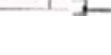

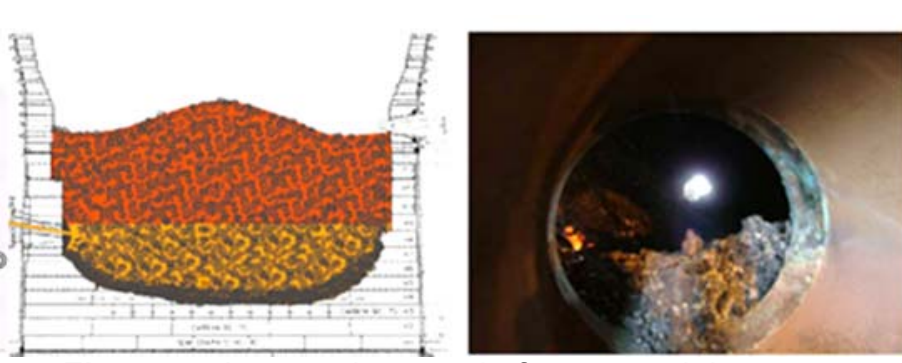

Figura 2. Aspecto interno do forno, parcialmente vazio, após a conclusão do Blowdown. [6]

Entretanto, a realização do Blowdown requer o planejamento e execução de procedimentos imprescindíveis para assegurar o seu êxito e também as devidas condições para a reforma e retorno de operação do alto forno.

\subsection{Planejamento Operacional}

Em termos operacionais, antes do Blowdown, deve-se buscar a máxima remoção de cascões das paredes do forno, assegurando a segurança e performance das atividades posteriores ao Blowdown. [6]

Desta forma, a fim de evitar a formação de cascão ou remover cascões presentes, pode-se realizar algumas alterações operacionais nos dias antecedentes ao Blowdown $[3,6-8,10]$, dentre elas:

i. Elevar a Marcha Periférica (maior fluxo de gás e calor nas paredes):

a) Reduzir 10 a $15 \%$ da velocidade de sopro pelas Ventaneiras para diminuir profundidade do Raceway (mais próximo à parede) e também melhorar eficiência de reação (contato gás $x$ carga);

b) Alterar distribuição de carga de modo a diminuir a relação Minério/Coque na parede, garantindo maior permeabilidade a passagem dos gases na parede.

ii. Aumentar a permeabilidade do Cadinho (maior temperatura do Fundo e Paredes do Cadinho para maior circulação de líquidos e remoção de material): 
a) Reduzir 10 a $15 \%$ da Basicidade da escória, cerca de 10 dias antes, (adição de Quartzo ou redução da basicidade na carga metálica), visando reduzir a temperatura de obtenção de líquidos e reduzir a quantidade de Álcalis e Zinco no interior do forno (diminuir formação de cascões);

b) Diminuição do $\% \mathrm{Al}_{2} \mathrm{O}_{3}$ na escória (menor Temperatura de Líquidos);

c) Aumento do $\% \mathrm{Mn}$ no Gusa (visar cerca de 0,7 a $0,9 \% \mathrm{Mn} 3$ a 5 dias antes) que permite aumento de fluidez do gusa uma vez que o óxido $\mathrm{MnO}$ formado reage com o carbono do Homem Morto $(\mathrm{MnO}+\mathrm{C}=\mathrm{Mn}+\mathrm{CO})$, incorporando manganês ao gusa e facilitando a fusão de escória/metal solidificados no cadinho (menor Temperatura de Líquidos);

d) Reduzir a refrigeração do cadinho (maior nível térmico para fundir material solidificado sob as paredes do cadinho);

e) Carregar coque de maior granulometria (trocar malhas de peneiras), melhorar qualidade do Coque (CSR, DI, etc) e cortar consumo de Small Coque;

f) Cortar $\mathrm{PCl}$ entre 1 a 3 dias antes, com CR 10 a 20\% superior à operação normal de All Coke, possibilitando maior energia para fusão dos materiais e também garantindo mais coque no cadinho (menos finos de carvão);

g) Trabalhar com DCl (Deadman Cleanliness Index - Índice de Limpeza do Cadinho) mais baixo (cadinho mais permeável e maior circulação de líquidos).

É importante salientar, no entanto, que o aumento da marcha periférica deve ser executado de forma gradual para não acarretar em queda abrupta de cascão que pode causar perda de nível térmico ou até deslocamento/furo de ventaneiras/caixas, com possibilidade de entrada de água no forno, ocasionando até mesmo resfriamento do forno ou "Marcha Fria" ("Chilled Hearth"). [7,10,11]

\subsection{Preparação de Equipamentos}

Algumas atividades são realizadas antes do Blowdown para preparar/fornecer os equipamentos vitais para este processo. Dentre as principais atividades, tem-se:

a) Furações dos pontos de injeção de $\mathrm{N}_{2}$ ao longo da cuba do forno (acionados durante o abaixamento da carga para diluição/purga dos gases);

b) Instalação e calibração das tomadas especiais para amostragem do gás do topo (controlar composição quando bleeders abertos no final do Blowdown);

c) Testar performance dos principais equipamentos do Topo;

d) Preparar um sistema específico de acompanhamento e controle do Processo de Blowdown, sumarizando os principais parâmetros de controle. [8, 10]

\subsection{Parada Pré-Blowdown}

Normalmente, antes do Blowdown, realiza-se uma parada específica do forno, por cerca de $8 \mathrm{~h}$, para a execução de certas atividades importantes [4, 10, 11], tais como:

a) Instalar bicos especiais de spray do topo (spray nozzles) que geram uma névoa de pequenas gotículas de água (droplets) de maneira a evitar o contato direto com o coque incandescente ou que a água escoe pelas paredes do forno (risco de elevação abrupta do \% $\mathrm{H}_{2}$ do topo ou até explosões se há $\mathrm{O}_{2}$ );

b) Aumentar a refrigeração da caixa de engrenagem do topo (preservação);

c) Limpar e acionar os pontos de injeção de $\mathrm{N}_{2}$ instalados ao longo da Cuba;

d) Até esta parada, ter concluído esvaziamento dos silos que não serão utilizados durante o Blowdown (small coque, fundentes, minério granulado). 


\subsection{Procedimento de Blowdown}

Após a Parada Pré-Blowdown, retorna-se a operação do forno, porém limitando o volume de sopro entre 60 a $80 \%$ do normal de operação (controle do gás de topo). Como o forno está em condição atípica (nível de carga baixo, Coque Rate elevado, volume de sopro reduzido, sem $\mathrm{PCl}$ e \%EO2) observam-se grandes mudanças na análise do gás do topo durante o abaixamento de carga. $[5,6,10]$

Inicialmente, o \%CO no gás de topo apresenta-se ligeiramente acima (maior $\mathrm{CO} / \mathrm{CO}_{2}$ ) devido elevado CR. Porém, durante o abaixamento de carga, o \%CO torna a diminuir mediante o aumento do $\% \mathrm{CO}_{2}$ devido à menor quantidade de coque em frente às ventaneiras, de maneira que haja menos carbono disponível para reagir com o $\mathrm{CO}_{2}$ (gerado no Raceway para formar o $\mathrm{CO}$ ). $[4,5,6,8,10]$

Em outras palavras, a reação de Boudouard (1.3) começa a perder eficiência (menor temperatura e disponibilidade de coque). E, como o carbono residual está sendo consumido pela reação 1.1 , a conversão do $\mathrm{CO}_{2}$ para $\mathrm{CO}$ deixa de ocorrer, permanecendo apenas a reação 1.1. [5,6]

$$
\begin{aligned}
& \mathrm{C}+\mathrm{O}_{2}=\mathrm{CO}_{2} \\
& 2 \mathrm{C}+\mathrm{O}_{2}=\mathrm{CO} \\
& \mathrm{C}+\mathrm{CO}_{2}=2 \mathrm{CO}
\end{aligned}
$$

Este aumento do $\% \mathrm{CO}_{2}$ ocorre até que $\% \mathrm{CO}$ e $\% \mathrm{CO}_{2}$ sejam iguais ( 10 a $15 \%$ de $\mathrm{CO}$ ou $\mathrm{CO}_{2}$ ), caracterizando-se o fenômeno chamado de "crossover" (cruzamento da composição do gás). Este é o principal indicativo da iminência de se concluir o Blowdown. $[6,8,10]$

Finalmente, $\mathrm{o} \% \mathrm{O}_{2}$ do topo aumenta para $1 \%$ a $2 \%$, porém, deve-se garantir $\% \mathrm{CO}+$ $\% \mathrm{H}_{2}<4 \%$ (limite de combustibilidade), nestes momentos finais $[5,6,8]$. Quando ocorrer o Crossover ou $0,8 \%$ de Oxigênio e $\% \mathrm{CO}+\% \mathrm{H}_{2}>4 \%$, o gás deve ser retirado pelos bleeders do topo, isolando o forno da rede de gás. [5,6,8]

Observando a carga no nível das ventaneiras e o colapso das mesmas, pode-se cortar o volume de sopro, concluindo o Blowdown. $[6,8,10]$

\subsection{Pós Blowdown}

Após concluir o Blowdown tem-se o apagamento propriamente dito do forno, o qual pode ser com todo material residual no cadinho ou, antes, pode-se realizar a Corrida de Salamandra (drenar parte de líquidos remanescentes). Para ambos tem-se o devido planejamento específico dos procedimentos $[1,5,6]$. Resumidamente, tem-se:

a) Corrida de Salamandra: preparação prévia dos furos (2 a 3 furos em alturas diferentes do cadinho), calha para direcionar líquidos para torpedos ou leitos preparados (secos/cobertos para evitar explosões) e garantir máxima fusão de materiais residuais no cadinho (preparação do Blowdown). [1,4,8,9,12]

b) Flood Quenching: resfriamento rápido do forno (26 a 36h) por meio do seu enchimento com água, até cobrir toda a carga remanescente após 0 Blowdown, necessitando menor prazo para acessar o interior do forno e iniciar serviços de limpeza, reparos e reforma do forno. $[8,9,12]$

c) Dry Quenching: resfriamento utilizado quando não há grande urgência para retorno de operação, utiliza $\mathrm{N}_{2}$ como agente refrigerante (mais lento que o Flooding), garantindo menor gradiente térmico aos refratários (fragilização e desgaste) e até evitando troca dos mesmos. [8,9,12] 


\section{MATERIAIS E MÉTODOS}

O trabalho visa apresentar uma descrição dos principais conceitos e procedimentos de realização do Blowdown de Altos Fornos, mostrando os resultados e discussões obtidos no procedimento de Blowdown do Alto Forno 03 da ArcelorMittal Tubarão, realizado no dia 10/11/2012.

Sendo assim, o procedimento Metodológico deste trabalho contempla basicamente:

a) Levantamento de informações e referências bibliográficas para embasamento;

b) Descrição da realização do procedimento de Blowdown;

c) Apresentação dos resultados obtidos e respectivas discussões.

Com isso, tem-se com este trabalho, fatos, dados de processo, características específicas (permitem melhor fundamentação), proporcionando, portanto, parâmetros de comparação de procedimentos distintos de Blowdown.

\section{RESULTADOS E DISCUSSÃO}

\subsection{Especificações do Alto Forno 03}

O Alto Forno N03 da ArcelorMittal Tubarão teve o seu início de operação (start-up) em 21/07/2007, permitindo uma capacidade de 2,8 Milhões de toneladas de gusa por ano [2]. As suas principais especificações estão citadas na Figura 3.

a)

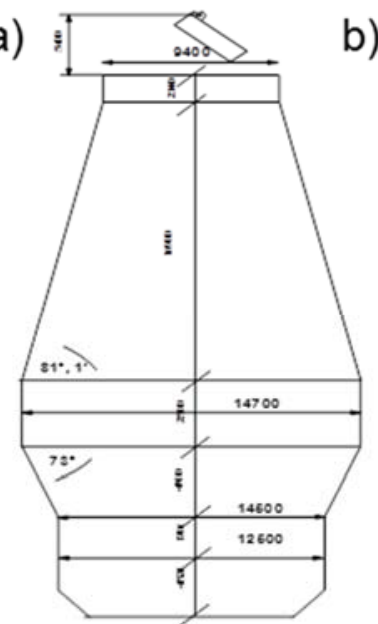

b)

\begin{tabular}{|c|c|}
\hline 1-ALTO FORNO & ESPECIFICACÖES \\
\hline Volume Interno (M) & $3.617 \mathrm{~m}^{3}$ \\
\hline Volume de Trabalho (VT) & $3,126 \mathrm{~m}^{3}$ \\
\hline Diâmetro do Cadinho & $125 \mathrm{~m}$ \\
\hline Furos de Gusa (FG) & 4 \\
\hline Ventaneiras (VAs) & 34 \\
\hline \multirow[b]{2}{*}{ Sistema de refrigeração } & $\begin{array}{c}\text { Tubos de refrigeraça ão (água } \\
\text { desmineralizada) }\end{array}$ \\
\hline & $\begin{array}{c}\text { Staves: } 5 \text { linhas com Stave de Cobre } \\
\text { no ventre e na Cuba inferior e o } \\
\text { restante de ferro fundido. }\end{array}$ \\
\hline 2-REGENERADORES & ESPECIFICACÖES \\
\hline Número & 3 Regeneradores \\
\hline Temperatura de Sopro (TS) & $1250^{\circ} \mathrm{C}$ \\
\hline 3-LIMPEZA DE GAS & ESPECIFICACÖES \\
\hline Balão coletor de Pó & Ciclone \\
\hline Scruber (PW) & 3 elementos do Bischoff \\
\hline 4-CARREGAMENTO & ESPECIFICACOES \\
\hline Tope & Tooo Bell-Less (calha rotativa) \\
\hline Sistema de carregam ento & Correia Transportadora \\
\hline Tremonhas do Tooo & 3 tremonhas de $60 \mathrm{~m} 3$ (baralelo) \\
\hline 5-PRESSAO DO TOPO & ESPECIFICACOES \\
\hline Tipo & Elementos do Bischoff \\
\hline & \\
\hline
\end{tabular}

Figura 3. Principais Especificações do Alto Forno 03 da ArcelorMittal Tubarão. a) Medidas do corpo do forno; b) Dados dos principais equipamentos. [2]

Com acumulado de 13,6 milhões toneladas de gusa, no dia 10/11/2012 foi realizado o Blowdown do AF3, conforme cronograma das principais atividades (Figura 4):

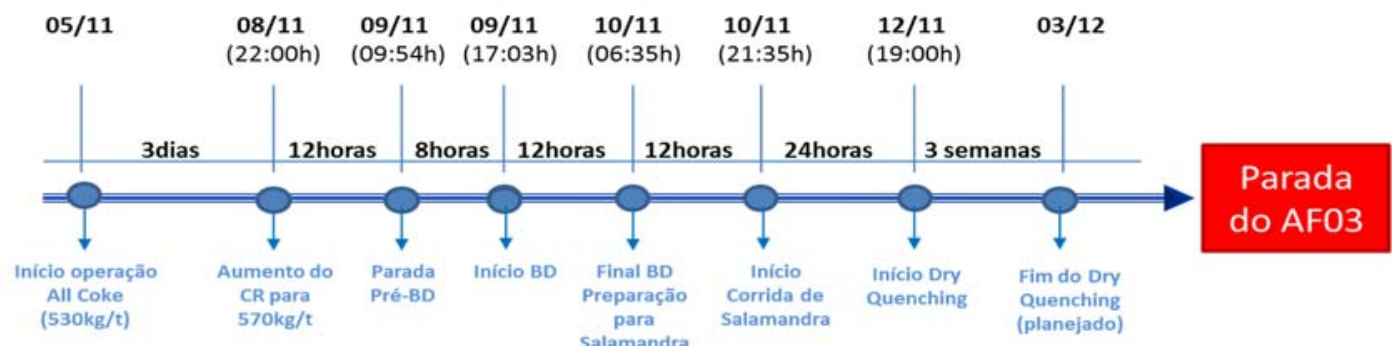

Figura 4. Cronograma de atividades realizadas durante Blowdown do Alto Forno 03. 


\subsection{Preparação do Blowdown}

\subsubsection{Programação do carregamento}

A preparação das cargas antes do Blowdown foram devidamente realizadas, sendo que as últimas cargas antes da parada pré-Blowdown foram:

- $70 \%$ de Sinter $+0 \%$ Minério Granulado $+30 \%$ de Pelota;

- B2 de 1,00 corrigido com quartzo;

- Silício visado: $1,0 \%$

- CR: $570 \mathrm{Kg} / \mathrm{tg}$

- Base de coque: 24 toneladas/carga

Com base nos cálculos realizados, o tempo total para a realização do Blowdown foi estimado para cerca de 18 horas (Figura 5), conforme plano de evolução do volume de sopro. A produção estimada foi de aproximadamente 1600 toneladas de gusa e 485 toneladas de escória (Slag Rate $=300 \mathrm{~kg} /$ tgusa), conforme Figura 6 .

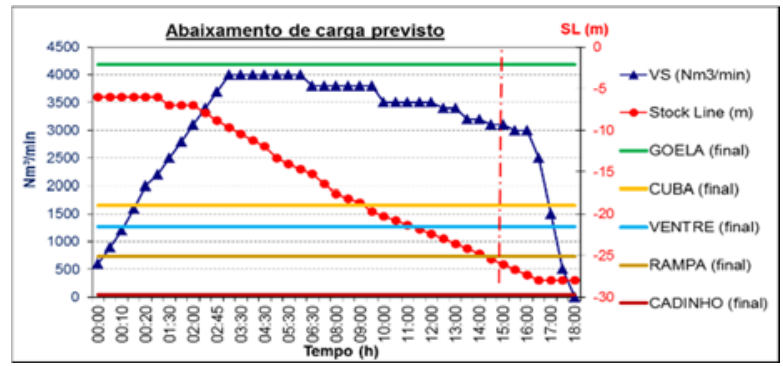

Figura 5. Abaixamento de carga até ao nível de ventaneiras conforme a vazão de sopro planejada.

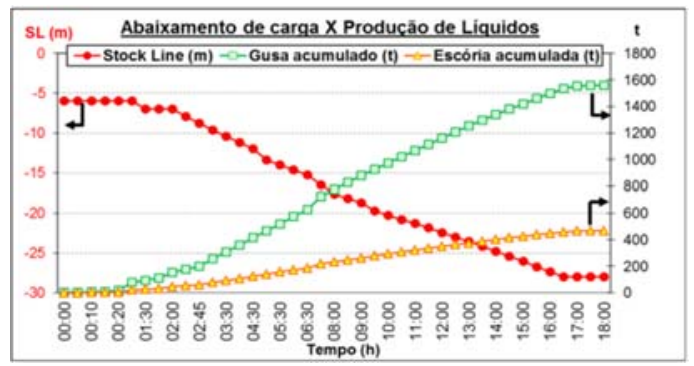

Figura 6. Cálculo de produção acumulada de gusa e escória durante este abaixamento.

\subsubsection{Aumento do fluxo de gás periférico}

Para adequada remoção de cascões, aumentou-se a temperatura das paredes do forno por meio de alterações de distribuição e elevação do CR (menor relação Minério/Coque), representada na Figura 7.

Deste modo, aumentou-se a Marcha Periférica (Figura 8), evidenciado pelo aumento do Índice de Fluxo Gasoso Periférico (IFGP: temperatura da sonda fixa medida no ponto 7 divida pela medição do ponto 5). A redução da velocidade do ar soprado pelas ventaneiras também permitiu esse aumento do IFGP (menor profundidade de sopro com fluxo mais próximo da parede). Em condições normais, a velocidade oscila em torno de 220 a $230 \mathrm{~m} / \mathrm{s}$ e a Figura 9 mostra que nos meses anteriores ao Blowdown, esta velocidade foi reduzida para aproximadamente 200 a $210 \mathrm{~m} / \mathrm{s}$.

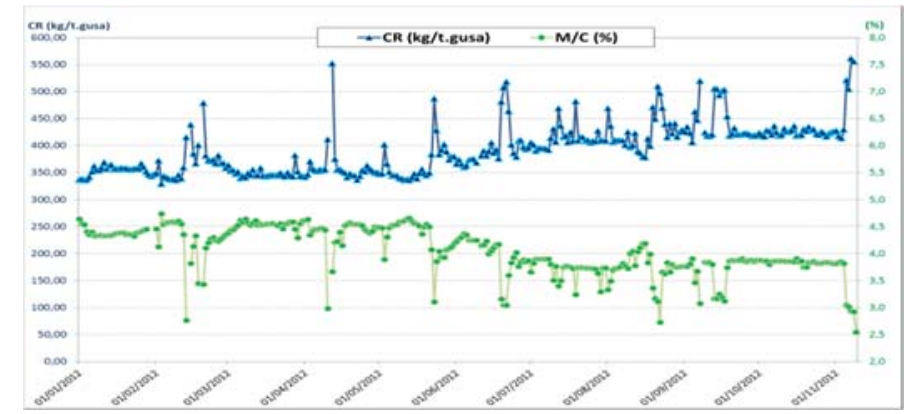

Figura 7. Elevação do Coque Rate aliada à diminuição da relação Minério/Coque. 


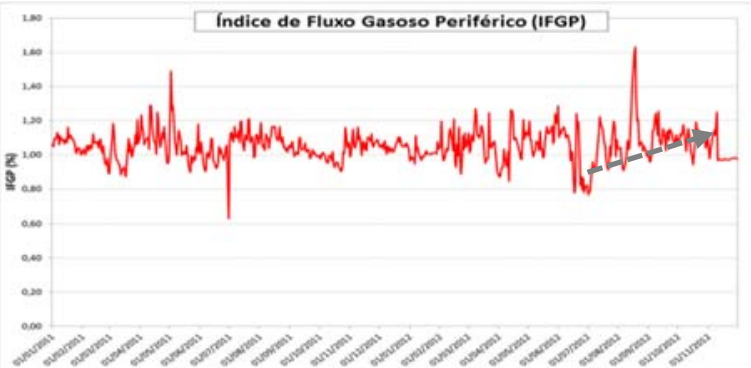

Figura 8. Aumento do Índice de Fluxo Gasoso Periférico (IFGP), comparando 2011 a 2012.

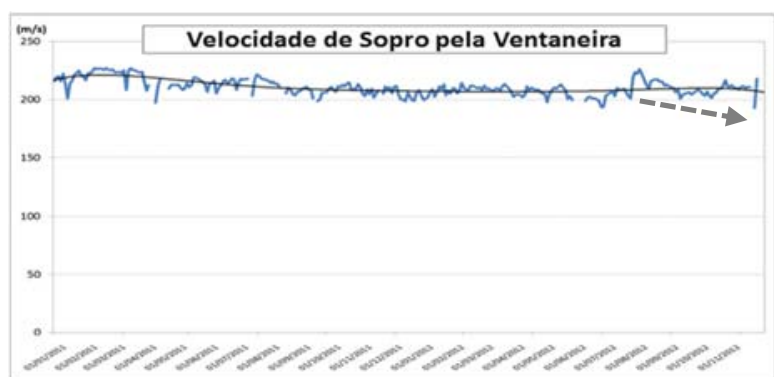

Figura 9. Redução da Velocidade do ar soprado pelas Ventaneiras (comparativo 2011 a 2012).

\subsubsection{Aumento de Permeabilidade do Cadinho}

Visando maior circulação de líquidos e limpeza do cadinho, o aumento da permeabilidade do cadinho se deu pelos seguintes procedimentos operacionais:

(a) Redução da refrigeração da soleira: 3 meses antes do Blowdown (17/08) reduziu-se a refrigeração para $25 \%$ do nominal $\left(300 \mathrm{~m}^{3} / \mathrm{h}\right)$ e a partir do dia 20/09 (2 meses antes), a vazão de água permaneceu praticamente zerada (Figura 10).

(b) Aumento do nível térmico do AF: a temperatura de gusa (operação normal: $1490^{\circ} \mathrm{C} \pm 10^{\circ} \mathrm{C}$ ) passou a ser visada em $1510^{\circ} \mathrm{C} \pm 10$ a partir do dia $01 / 11$ e a elevação do \%Si mostra o maior nível térmico (Figura 11).

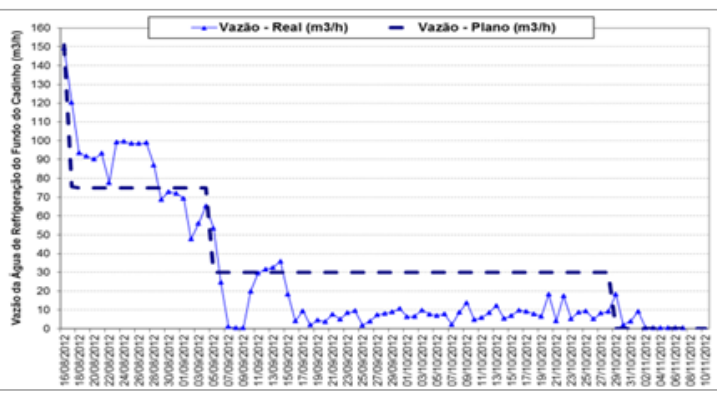

Figura 10. Redução da vazão de água de refrigeração do fundo do cadinho.

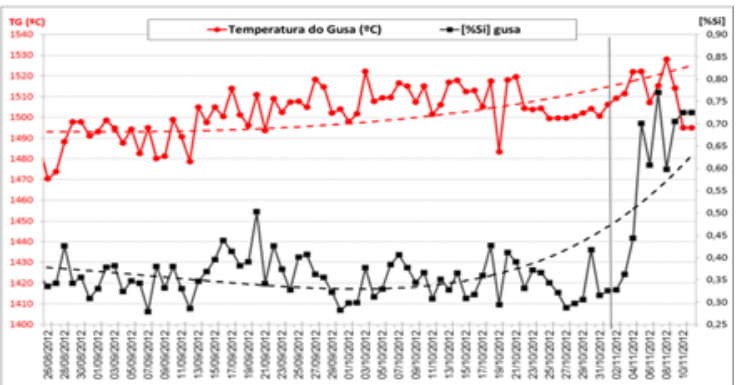

Figura 11. Aumento de temperatura de gusa e teor de silício do gusa.

(c) Reducão do \%Small Coque (SC): cessou-se o carregamento de Small Coque no dia 03/11 (Figura 12), permitindo maior permeabilidade (maior fração de vazios);

(d) Corte de carvão (PCl): foi realizado (menor \%fino enfornado), sendo necessária redução do Enriquecimento de Oxigênio (EO2) do ar soprado (Figura 13).

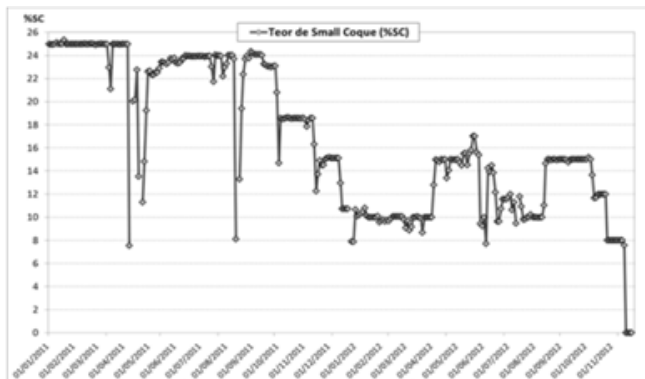

Figura 12. Redução do consumo de Small Coque (SC) para aumento da permeabilidade.

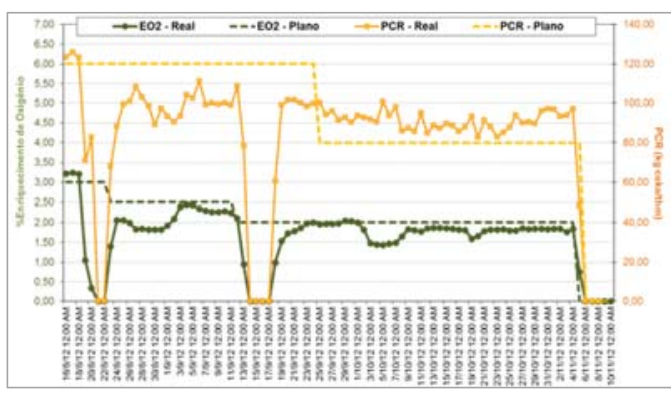

Figura 13. Redução de $\mathrm{PCl}$ e EO2. 
(e) Redução da basicidade da escória (B2): foi realizada através do controle do carregamento de Quartzo (Figura 14) e pela diminuição da Basicidade do Sinter devido ao aumento da participação de Sinter no Leito (Figura 15).

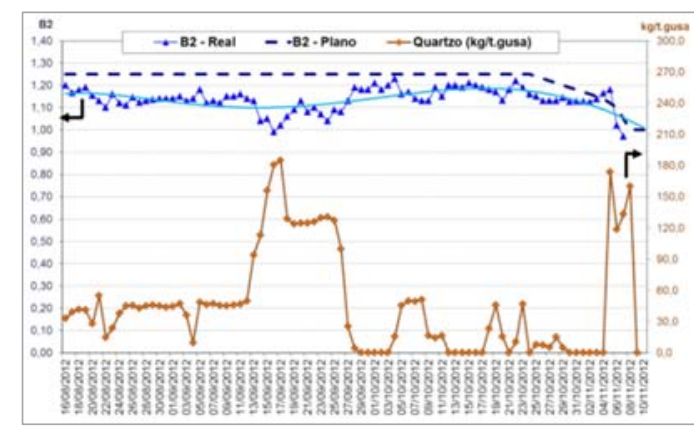

Figura 14. Aumento do consumo de Quartzo na última semana antes do Blowdown.

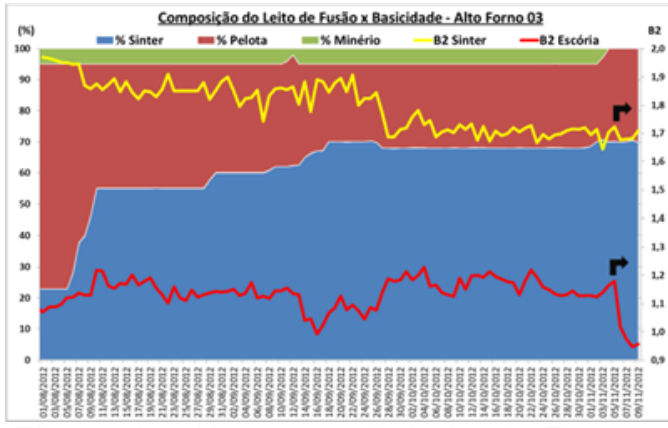

Figura 15. Alterações do Leito de Fusão e da B2 do Sinter para ajustar a B2 escória.

(f) Aumento do \%Mn no gusa: visando maior fluidez, aumentou-se o \%Mn via elevação do \%Mn no Sinter, maior participação de Sinter no Leito e aumento do nível térmico (maior incorporação de Mn), conforme mostrado na Figura 16. Para não impactar o AF1 e AF2, reduziu-se um pouco o \%Mn no Sinter de 0,80\% para $0,70 \%$ e carregou-se em torno de $120 \mathrm{t} / \mathrm{d}$ de minério granulado de alto $\% \mathrm{Mn}$ apenas no AF3 entre 23 a 31/10.

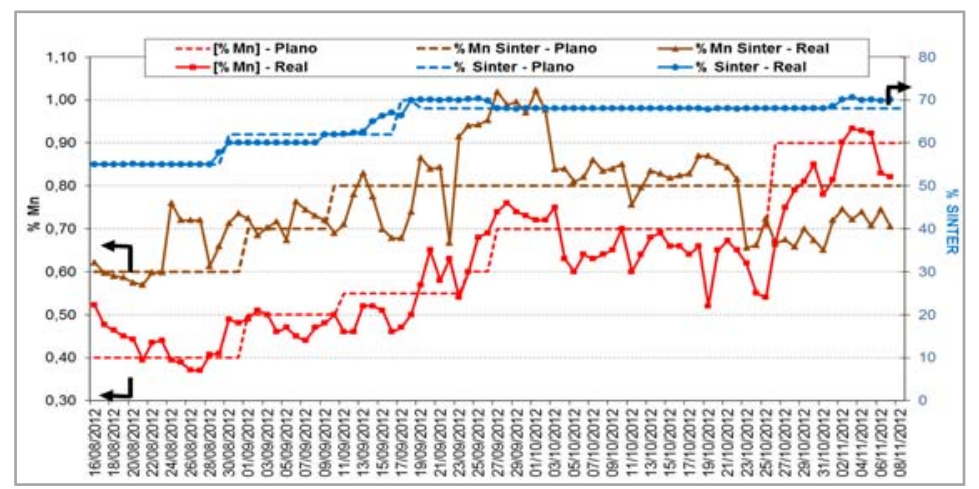

Figura 16. Elevação do \%Mn no gusa pelo aumento do \%Sinter no leito e do \%Mn no Sinter.

\subsubsection{Acompanhamento da temperatura do cadinho}

Realizou-se acompanhamento específico das temperaturas do Cadinho, tomando como base a temperatura do Fundo (THB02) para verificar o aumento ou diminuição do fluxo de líquidos (diminuir volume de material solidificado/inativo e facilitar Corrida de Salamandra), como mostrado na Figura 17.

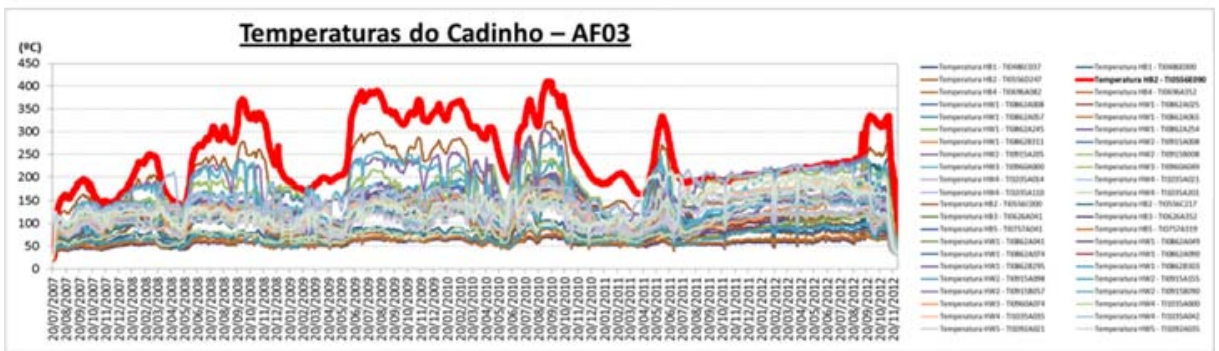

Figura 17. Histórico de temperaturas do cadinho do Alto Forno 03 durante toda a campanha. 
Primeiramente, foi feita a comparação do comportamento da Temperatura do Fundo do Cadinho relacionando com a Temperatura da Parede do Cadinho (Figura 18).
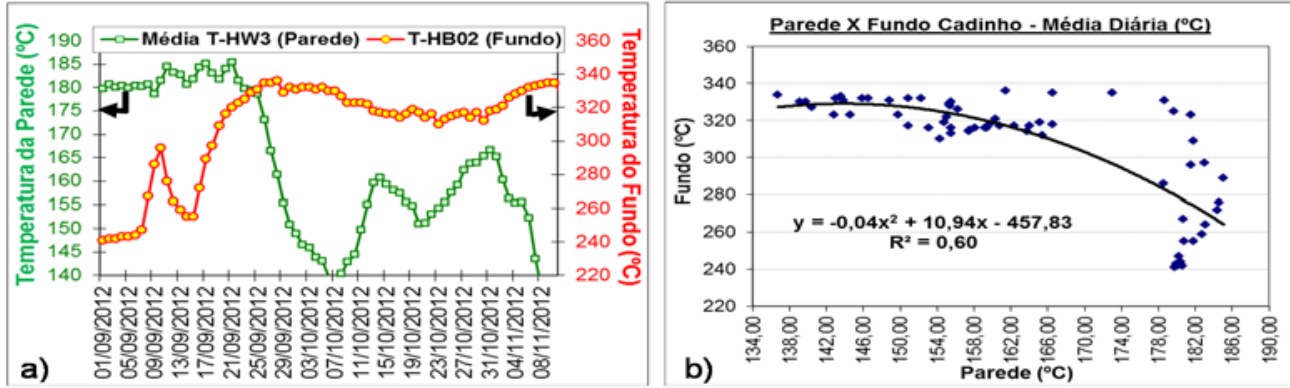

Figura 18. Comparação entre as temperaturas do Fundo (THB02) e da Parede (TW3): a) Acompanhamento; b) Correlação.

Assim, nota-se que os procedimentos adotados permitiram elevação da temperatura do Fundo (THB02: $240^{\circ} \mathrm{C}$ para $320^{\circ} \mathrm{C}$ ). Porém, a partir do dia $28 / 09$, o fundo voltou a decrescer enquanto a temperatura da Parede (TW3) que estava diminuindo, tornou a aumentar. Isto se deve a um período de perda de permeabilidade do Cadinho (maior compactação do Homem Morto), dificultando circulação de líquidos e diminuindo \%C incorporado ao gusa. Esta condição pode ser explicada pelo aumento do $\mathrm{DCl}$. Quando o $\mathrm{DCl}$ voltou a diminuir (maior circulação de líquidos), o Fundo voltou a elevar-se ocasionando maior incorporação de carbono (Figura 19).

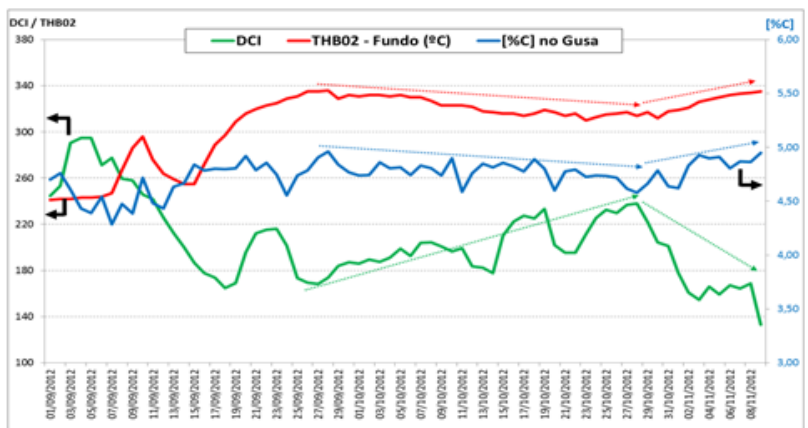

Figura 19. Comportamento da Temperatura do Fundo do Cadinho (THB02) em relação ao DCl, sendo o teor de carbono no gusa $(\% \mathrm{C})$ um indicativo do nível térmico e $\mathrm{DCl}$.

Para complementar, foram realizadas correlações para determinar quais fatores estiveram mais associados com o comportamento da Temperatura do Fundo do Cadinho (THB02) no período analisado, conforme Figura 20 e Tabela 1.

\begin{tabular}{|c|c|c|c|c|c|c|c|c|c|}
\hline & $\begin{array}{c}\text { THBO2 } \\
\left({ }^{\circ} \mathrm{C}\right)\end{array}$ & $\begin{array}{l}\text { YMn no } \\
\text { Gusa }\end{array}$ & $\mid(M n) / M n]$ & B2 & $\mid \begin{array}{c}\text { CSR (pós } \\
7 \text { dias) }\end{array}$ & IFGC & (\%A/2O3) & $\begin{array}{c}D I \text { (pós } 7 \\
\text { dias) }\end{array}$ & $\begin{array}{l}\text { Vazáo de Agua } \\
\text { Fundo do } \\
\text { Cadinho }\left(m^{1} / h\right)\end{array}$ \\
\hline THBO2 $\left({ }^{\circ} \mathrm{C}\right)$ & 1,0000 & & & & & & & & \\
\hline SMn no Gusa & 0.8726 & 1.0000 & & & & & & & \\
\hline$(M n) / M n]$ & .0 .8434 & 0.8524 & 1,0000 & & & & & & \\
\hline$B 2$ & -0.2511 & 0,1179 & $-0,3441$ & 1,0000 & & & & & \\
\hline CSR (pós 7 dias) & 0.5410 & 0.1771 & 0,1683 & $.0,7788$ & 1,0000 & & & & \\
\hline IFGC & 0.8951 & 0.8477 & -0.7284 & 0.2807 & -0.6380 & 1,0000 & & & \\
\hline (\%AN203) & $-0,7977$ & 0,0444 & $-0,3407$ & 0.6877 & $-0,9671$ & 0.2912 & 1,0000 & & \\
\hline DI (pós 7 dias) & 0,7771 & 0.6392 & -0.2990 & $-0,0254$ & 0,0976 & 0,9076 & 0,1421 & 1,0000 & \\
\hline Vazão de Água Cadin & .09324 & .0 .7609 & 0.6635 & 0.0855 & .0 .5103 & .0 .8280 & 0.2030 & $-0,7806$ & 1,0000 \\
\hline
\end{tabular}

Figura 20. Resultados obtidos com as correlações entre os parâmetros operacionais e a temperatura do Fundo do Cadinho (THB02). 
Tabela 1. Resultado sumarizado, apresentando o grau de correlação das variáveis (R2) com a Temperatura do Fundo do Cadinho (THB02).

\begin{tabular}{lc}
\multicolumn{1}{c}{ Variável } & $\mathbf{R}^{\mathbf{2}}$ \\
\hline IFGC (Marcha Central) & $\mathbf{0 , 9 0}$ \\
\hline$[\% \mathrm{Mn}]$ no Gusa & $\mathbf{0 , 8 7}$ \\
\hline DI do Coque & $\mathbf{0 , 7 8}$ \\
\hline CSR do Coque & $\mathbf{0 , 5 4}$ \\
\hline Vazão de Água no fundo do Cadinho $\left(\mathrm{m}^{3} / \mathrm{h}\right)$ & $-0,93$ \\
\hline$(\mathrm{Mn})_{\text {esc }} /[\mathrm{Mn}]$ gusa & $-0,84$ \\
\hline$\left(\% \mathrm{Al}_{2} \mathrm{O}_{3}\right)$ da escória & $-0,80$ \\
\hline $\mathrm{B} 2$ da escória & $-\mathbf{0 , 2 5}$ \\
\hline
\end{tabular}

Desta forma, no levantamento realizado, os parâmetros que apresentaram maior correlação com o aumento da temperatura do cadinho foram:

a) Redução da vazão da água para refrigeração do Fundo do Cadinho, possibilitando menor troca de calor e perda térmica do cadinho;

b) Aumento da Marcha Central (IFGC), permitindo maior circulação dos líquidos, principalmente em direção ao fundo do cadinho;

c) Aumento do [\%Mn] do gusa, aumentando fluidez dos líquidos;

d) Redução da relação (Mn)escória/[Mn]gusa atrelada à redução de arriamentos e chegada de material mal preparado (não reduzido) ao cadinho;

e) Aumento dos parâmetros de qualidade do coque (DI e CSR) a fim de amenizar a entrada/geração de finos (permeabilidade do cadinho);

f) Diminuição de $\% \mathrm{Al}_{2} \mathrm{O}_{3}$ e $\mathrm{B} 2$ da escória (menor temperatura para formação de líquidos), garantindo menor perda de calor para fusão da escória.

\subsection{Parada Pré-Blowdown}

A Parada Pré-Blowdown, foi realizada no dia 09/11/12 às 09h54 (duração de 07h09min) com nível de carga em torno de 9,0m (Figura 21). Antes da parada, carregou-se $100 \mathrm{t}$ de coque extra (5 cargas) umectados na correia principal para amenizar elevação da TGT (Figura 22). Além disso, também foi alterado o limite de abertura automática dos sprays do topo de $250^{\circ} \mathrm{C}$ para $320^{\circ} \mathrm{C}\left(1^{\circ}\right.$ estágio: $\left.80 \mathrm{t} / \mathrm{h}\right)$ e de $270^{\circ} \mathrm{C}$ para $350^{\circ} \mathrm{C}\left(2^{\circ}\right.$ estágio: $\left.160 \mathrm{t} / \mathrm{h}\right)$.

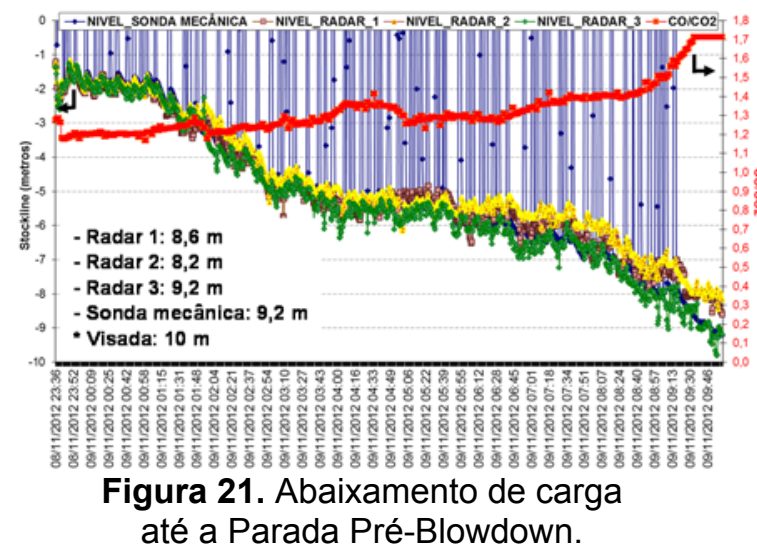

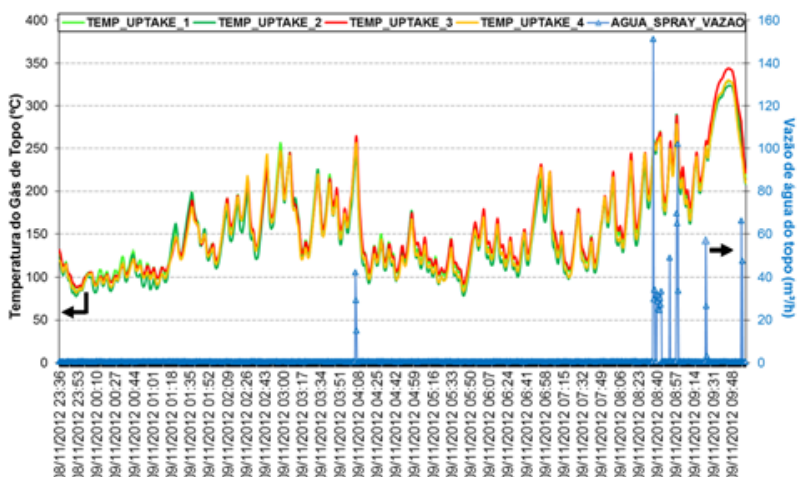

Figura 22. Evolução da temperatura do topo e vazão de água dos sprays antes da Parada. 
As principais atividades durante esta parada foram a instalação dos bicos de sprays especiais e a conexão das linhas de $\mathrm{N}_{2}$ da cuba. Antes de retornar o AF3, verificouse também o funcionamento do sistema de sprays, dos bleeders, da refrigeração da caixa de engrenagem e a calha rotativa ficou operando no anel mais externo (anel 15) a fim de evitar aquecimento localizado da calha, preservando o equipamento.

\subsection{Procedimento de Blowdown}

O retorno do AF3 se deu às $17 \mathrm{~h} 03$ do dia 09/11 e a descida de carga transcorreu normalmente conforme controle dos parâmetros (Figura 23). Assim, foi elevada a Vazão de Sopro (VS) para $4100 \mathrm{Nm}^{3} / \mathrm{min}$ (75\% do VS normal).

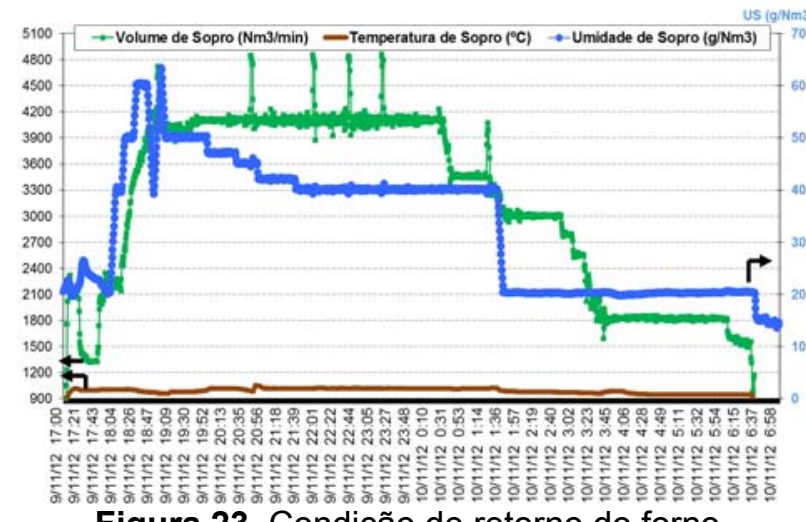

Figura 23. Condição de retorno do forno após Parada Pré-Blowdown.

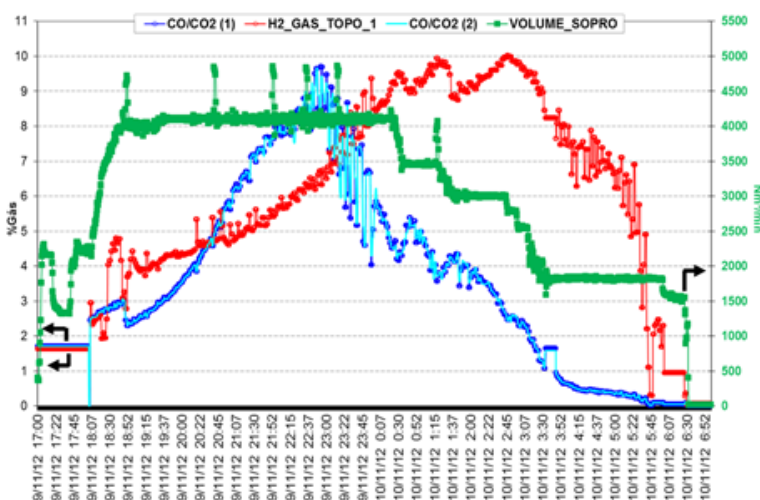

Figura 24. Comportamento do gás de topo aliado à redução do Volume de Sopro.

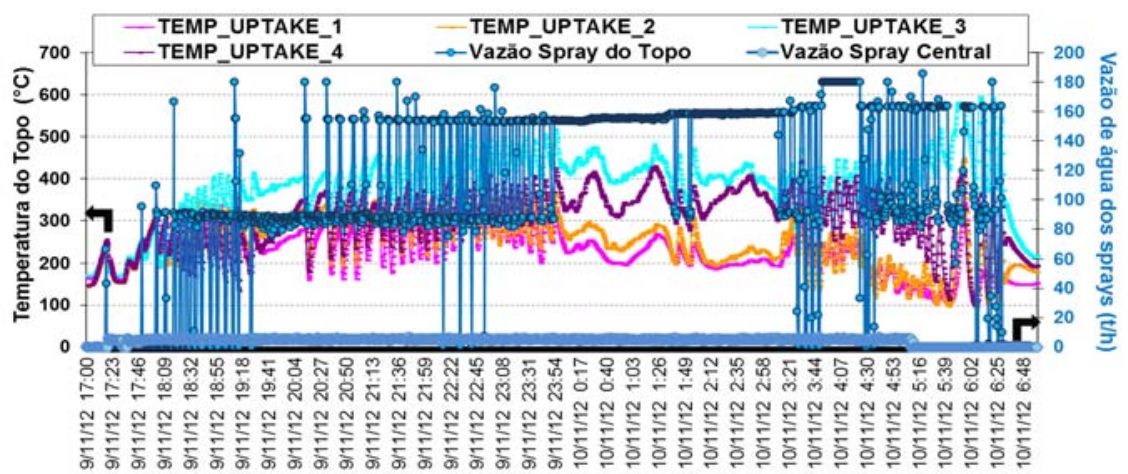

Figura 25. Controle da Temperatura do Gás de Topo pela vazão dos sprays de água do Topo.

Com a elevação da TGT, foi necessário reduzir VS até $3500 \mathrm{~nm} 3 / \mathrm{min}$ ás $00 \mathrm{~h} 39$ quando se tinha $12 \% \mathrm{H}_{2}$ no topo (Figura 24). A TGT também foi controlada pela vazão de água dos sprays do topo (Figura 25) e a medida que o nível de carga atingia o nível dos pontos de $\mathrm{N}_{2}$ da cuba, foram sendo abertos os respectivos anéis de $\mathrm{N}_{2}\left(3000 \mathrm{Nm}^{3} / \mathrm{h}\right.$ para cada um dos 3 anéis).

Às $01 \mathrm{~h} 18$ foi cortada a combustão dos regeneradores devido baixo $\mathrm{PCl}$ do gás (elevada presença de vapor/condensado). Por isso, reduziu-se VS para 3000 $\mathrm{nm}^{3} /$ min e cortada Umidade de Sopro.

Quando os níveis de carga atingiram 23 metros, a TGT ainda estava muito alta, sendo necessário reduzir a VS para $2800 \mathrm{~nm} 3 / \mathrm{min}$. Procedeu-se o processo de isolar o AF3 da rede de gás da usina (Utilidades), forçando-o a ser queimado pela torre. Devido capacidade de queima da Torre, reduziu-se VS para $2500 \mathrm{~nm}^{3} / \mathrm{min}$. 
Às 03h51, quando o nível de carga estava em $24 \mathrm{~m}$ ( $1,7 \mathrm{~m}$ acima das ventaneiras), o $\% \mathrm{CO}$ do topo diminuiu até ocorrer o Crossover $\left(\% \mathrm{CO}=\% \mathrm{CO}_{2}\right)$, ou seja, com o abaixamento da carga e o consumo de coque sem reposição pelo topo, chegou-se a uma condição de deficiência de coque para promover a geração de gás redutor (CO) por meio da reação de gaseificação do coque pelo $\mathrm{CO}_{2}$ (Reação de Boudouard). Logo, o coque remanescente reagia com o $\mathrm{O}_{2}$ formando $\mathrm{CO}_{2}$, porém este $\mathrm{CO}_{2}$ não era convertido em $\mathrm{CO}$ devido ao esgotamento do coque em frente às ventaneiras. Sendo assim, observou-se a elevação do $\% \mathrm{CO}_{2}$ e diminuição do $\% \mathrm{CO}$ no gás de topo (Figura 26). Logo, foi aberta corrida paralela como procedimento de parada.

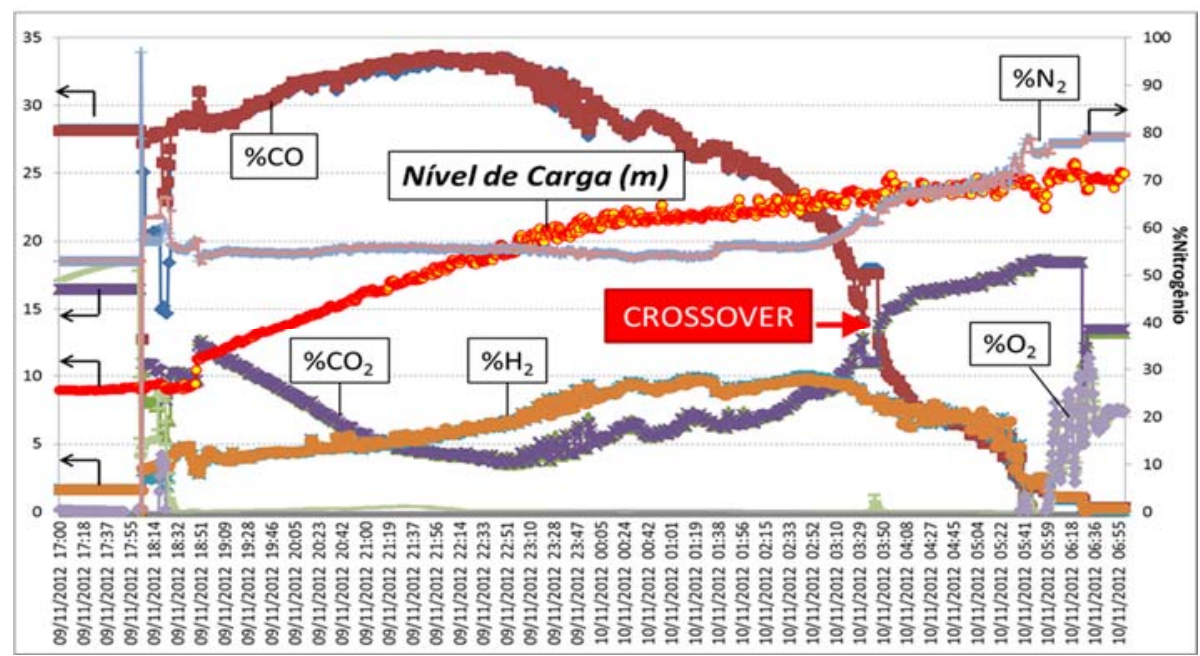

Figura 26. Comportamento do gás de topo e nível de carga durante Blowdown, identificando o momento do Crossover.

Às $05 \mathrm{~h} 44$ a soma de $\mathrm{CO}+\mathrm{H}_{2}$ estava inferior a $4 \%$, mas o Oxigênio não subiu. Como continuava a adição de água no topo para controlar a TGT, reduziu-se o VS para $1500 \mathrm{Nm}^{3} / \mathrm{min}$. minimizando entrada de água pelo topo.

As primeiras ventaneiras começaram a entrar em colapso às $06 \mathrm{~h} 05$ quando $\circ \mathrm{O}_{2}$ subiu para $1,2 \%$ (menos coque disponível para reagir com $\mathrm{O}_{2}$ ).

A conclusão do Blowdown (corte de sopro) ocorreu às 06h35 do dia 10/11/2012 (Figura 27), quando foi aberto o air bleeder (Backdraft) e Snort.

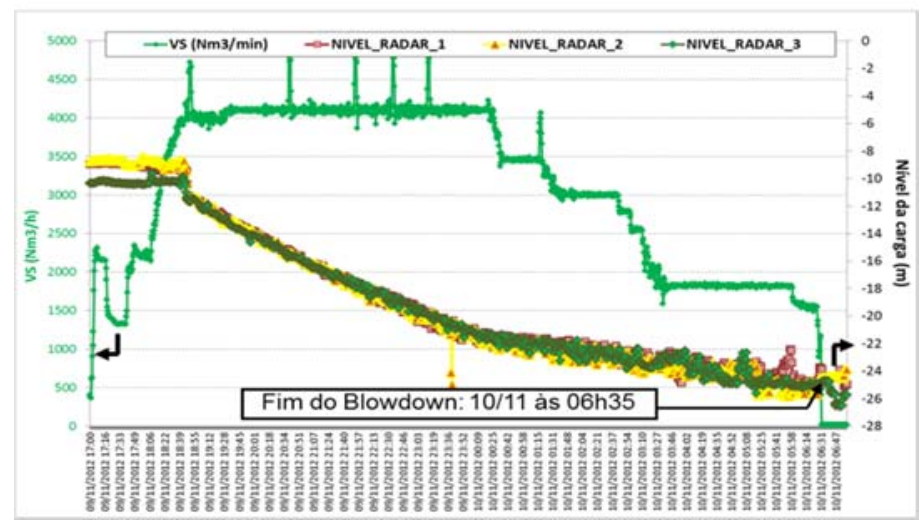

Figura 27. Abaixamento do Nível de Carga até o Nível das Ventaneiras e corte do sopro (VS).

O tempo total do Blowdown foi de 13h15min, após retorno da parada Pré-Blowdown. 


\subsection{Procedimento Pós-Blowdown}

O procedimento de perfuração foi realizado e ocorreu a corrida da Salamandra às $21 \mathrm{~h} 43(10 / 11)$ no $2^{\circ}$ Furo (não obteve-se êxito no $1^{\circ}$ furo) com um total de $115 \mathrm{t}$ de material para o pátio, apresentando composição química conforme a Tabela 2.

Tabela 2. Resultados de amostragem da Composição Química da Salamandra.

\begin{tabular}{ccc}
\hline Composição & $\mathbf{1}^{\mathbf{a}}$ Amostragem & $\mathbf{2}^{\mathbf{a}}$ Amostragem \\
\hline \%C & 4,45 & 4,63 \\
$\mathbf{\%} \mathbf{S}$ & 1,27 & 1,17 \\
$\mathbf{M n}$ & 1,05 & 1,00 \\
$\mathbf{\%}$ & 0,027 & 0,027 \\
$\mathbf{\%} \mathbf{T i}$ & 0,07 & 0,065 \\
\hline
\end{tabular}

Não houve nenhum incidente e o furo foi fechado ás 01 h10 do dia 11/11/12.

Após o término de todo o processo de Blowdown, Salamandra e Quenching do Alto Forno 03 da ArcelorMittal Tubarão, foi possível observar a eficiente remoção de cascões das Paredes, o abaixamento de carga até às ventaneiras, o esgotamento de coque em frente às ventaneiras e o aspecto do Homem Morto residual, como mostrado na Figura 28.
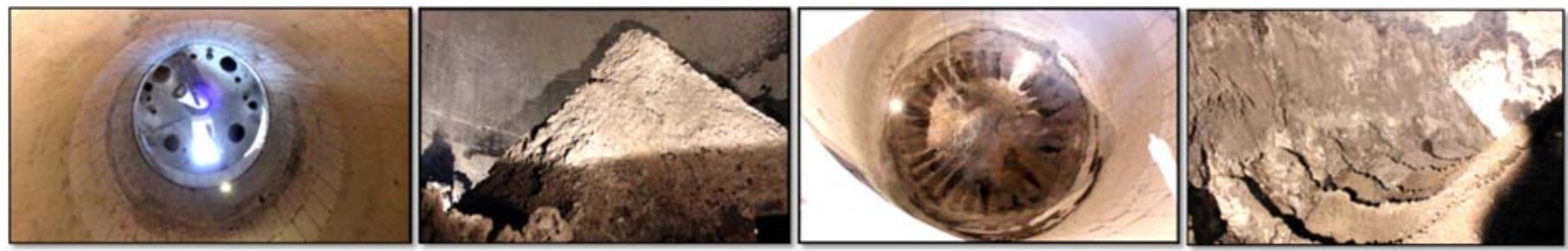

Figura 28. Aspecto interno do Alto Forno 03 após todos os procedimentos referentes ao Blowdown.

\section{CONCLUSÃO}

O Blowdown consiste no desligamento do de um alto forno por meio do abaixamento de carga até o nível das ventaneiras. Os resultados obtidos demonstraram como se dá este mecanismo, ratificando que o planejamento e acompanhamento do processo são determinantes durante o desempenho do Blowdown.

Outrossim, os resultados alcançados mostraram que o na preparação do AF03, o aumento da marcha central, [\%Mn] no gusa, CSR e DI do Coque, além da redução da refrigeração do fundo do cadinho, redução da $\% \mathrm{Al}_{2} \mathrm{O}_{3}$ e $\mathrm{B} 2$ da escória, foram os parâmetros que apresentaram maior correlação, ou seja, acompanharam o comportamento de elevação da temperatura do fundo do cadinho (permeabilidade).

Com o término do Blowdown, constatou-se que o AF03 apresentou excelentes condições internas (bom esgotamento e paredes livres de cascões).

Portanto, em meio às situações adversas que o mercado siderúrgico possa propiciar aliado à vida útil dos Altos Fornos, nota-se a importância do conhecimento técnico sobre o Blowdown na operação dos Altos Fornos.

\section{Agradecimentos}

A Deus, a ArcelorMittal Tubarão, aos familiares e amigos. 


\section{REFERÊNCIAS}

$1 \quad$ Araujo LA de. Manual de Siderurgia. São Paulo: Arte e Ciência; 1997.

2 ArcelorMittal Tubarão. Ficha Técnica Alto Forno 03. Fluxo de produção. [acesso em 20 mar. 2013]. Disponível em:

http://www.cst.com.br/usina/fluxo_producao/popfluxo.htm.

3 Bitsadze GA, Parastashvili VV, Kobakhidze IA, Khoshtariya SF. Formation of scab and its removal from Blast-Furnace under operating-conditions of Rustavi ironworks and steelworks. Steel in the USSR. 1987. 17(1): 352-355.

4 Burgo JA. The manufacture of pig iron in the blast furnace.11.Pittsburgh: The AISE Steel Foundation; 1999.

5 Geerdes M, Toxopeus HVV, Van der Vliet C. Modern Blast Furnace Ironmaking: an introduction. 2. Amsterdam: IOS Press; 2009.

6 Geerdes M, Spiering T, Wise J, Gravemaker GJ. Blast Furnace Blow-down minimizing environmental emissions. AISTech. 2009.1: 63-67.

7 Raipala K. On Hearth phenomena and hot metal carbon content in Blast Furnace. Finlândia. Tese [Doutorado em Ciência e Tecnologia] - Departamento de Ciência dos Materiais e Metalurgia - Universidade de Tecnologia de Helsinki; 2003.

8 Ricketts JA. A. Blowdown: Preparation, Process, Salamander, Quenching. ArcelorMittal. 2011. 1-170.

9 Ricketts JA. Flooding Quench. ArcelorMittal. 2013. 1-56.

10 Rorick FC. Challenging Blast Furnace Operations: Blast Furnace Ironmaking Course. McMaster University. 1999. 2(14): 1-61

11 Strassburger JH, Brown DC, Dancy TE, Stephenson RL. Blast Furnace: Theory and Practice. 2. Nova lorque: Gordon and Breach Science Publishers; 1984. 537-646.

12 Van Laar RJ, Spiering TA. Tapping the Blast Furnace Salamander. Millennium Steel: Raw Materials and Ironmaking. 2005. 1: 63 - 67. 